\title{
Double T Filter Based New Cable Insulation Detection Approach And Design for the Subway Train
}

\author{
Peng tian ${ }^{1, a^{*}}$, Decun dong ${ }^{1, b}$ and Hongliang $\operatorname{Pan}^{2, c}$ \\ ${ }^{1}$ School of Transportation Engineering Tongji University in City Shanghai \\ ${ }^{2}$ The Technology Research Center of Magnetic Traffic Engineering in City Shanghai \\ atianpeng890803@163.com, bddc@tongji.edu.cn, ${ }^{c}$ panhongliang@tongji.edu.cn
}

Keywords:The subway equipment;cable insulation detection;double $\mathrm{T}$ filter;Electromagnetic interference

\begin{abstract}
On-board cable in the subway is an essential competent in the whole power system for the electric transmission, power allocation and signal control. The cable insulation condition plays an important role in safe and reliable operation of the subway. Traditional insulation detection devices haven't taken into account the impact of the electromagnetic interference on insulation test detection. Severe electromagnetic interference would impact the unstable operation and the data volatility of the online monitoring system. Therefore, the paper proposes a double $\mathrm{T}$ filter based new approach to cable insulation detection in the subway. The proposed approach can not only improve the detection accuracy of the cable insulation, but can also fit with complex noise signal interference environment in the subway. With the proposed approach, stability of equipment operation can be guaranteed.
\end{abstract}

\section{The Necessity of Electric Cable Insulation Measurement in the Subway}

Today's subway is complete in almost every function, and be equipped with various electrical equipment. Therefore more cables are laid correspondingly. The train body is mainly made of stainless steel and aluminum alloy. Once cables are scratched or broken, electricity leakage will occur and take serious effects for the train operation and passengers' safety. Additionally, when the cable insulation is damaged, the ground fault will occur, lead to the faulty operation of signal circuits and control circuits, or even result in serious consequences such as fire hazard and equipment damage. More seriously, these effects will break the normal operation of the whole system, and even cause casualties.

According to the statistics, $60 \%$ to $80 \%$ of the accidents are cable insulation driven malfunction among the operation of the power equipment in subway. In order to guarantee passengers' safety and running security, cable insulation detection must be conducted.

In urban rail transit systems, there are many necessary equipment and piping systems for normal operation .Among these devices, some may produce strong electromagnetic interference during the operation. These devices include overhead traction wires, leakage coaxial cable, mobile communication modules, etc. Some testing equipment is more sensitive to electromagnetic interference. This device can't work normally or even lead to mis-operation if affected by electromagnetic interference. It will bring serious consequences to the safe operation of rail transit system. 
The traditional design of cable insulation detection concept fails to go through the field noise filter processing. However, because of the complexity of on-board power grid and operating environment in subway train, the noise interference is a necessary considered factor to improve the precision of the cable insulation detection. Based on traditional experiences, there are few researches about precise insulation detection in the complex noise interference the environment of the subway train. Therefore the high precise cable insulation detection approach is particularly important for complex noise interference environment in the subway train.

\section{Double T Filter Based New Cable Insulation Detection Hardware Design for the Subway Train}

There are four modules in cable insulation detection hardware design for the subway train .They are power supply module, insulation resistance sampling module, the AD conversion module and the insulation resistance data processing module. Double $\mathrm{T}$ filter circuit is integrated into the insulation resistance sampling module. Insulation resistance sampling module is shown in figure 1.

\section{Insulation Resistance Sampling Module}

The diagram of the sampling module circuit of insulation resistance is shown in Fig 1.

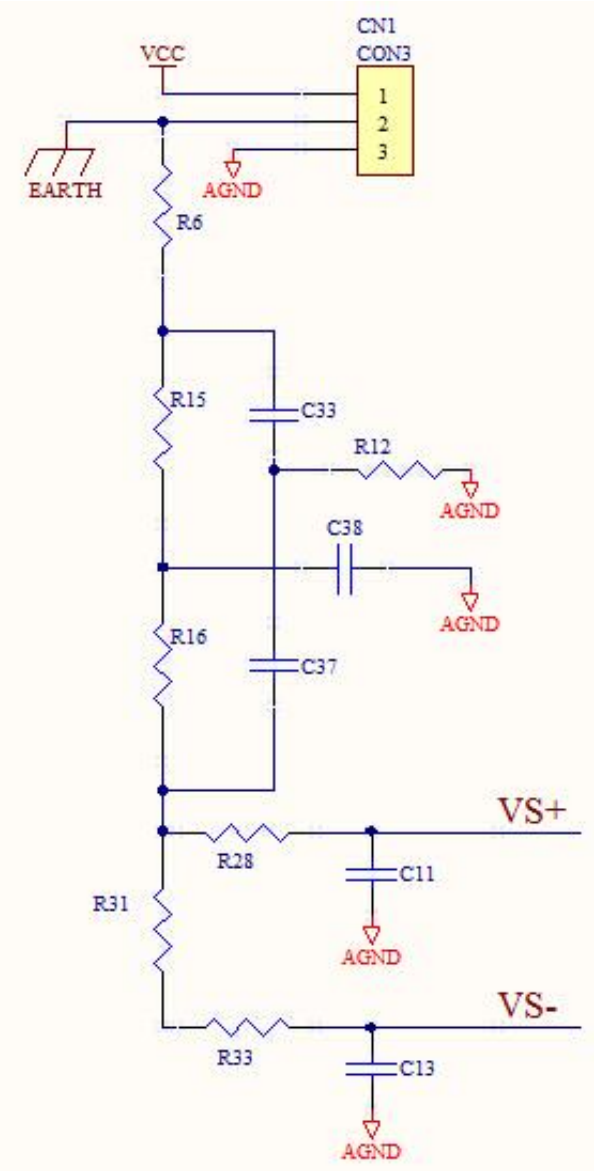

Fig. 1 The sampling module circuit of insulation resistance

Where, the 2nd pin of CN1 connects to the power supply VCC (external test cable) and the 3nd pin of $\mathrm{CN} 1$ connects to the earth. VS + and VS- are the sampling voltage signal.

In the subway train operating environment, there is serious electromagnetic interference which disturbing insulation detection system .In order to eliminate the measurement error produced by $50 \mathrm{~Hz}$ noise jamming, we combine double $\mathrm{T}$ filter circle with insulation detection system. 


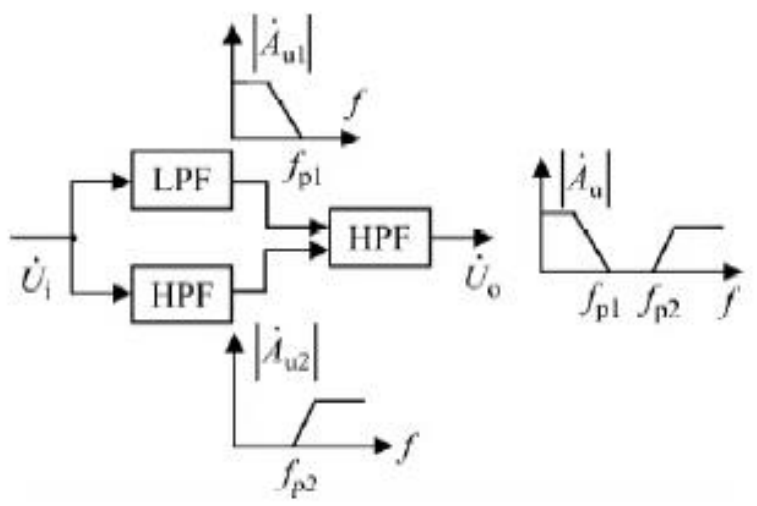

Fig.2 Block diagram of band-stop filter

In the signal processing circuits, it is often to use the band stop filter blocking interference signals fleeing. As shown in Fig 2, band-stop filter usually use a low-pass filter (LPF) and a high-pass filter (HPF) in parallel. The cutoff frequency, fp1, of the low pass filter should be lower than the cutoff frequency, fp2, of high-pass filter. The value of the stop band is (fp2 - fp1).Band-stop filter can constitute a noise-squelch circuit which can have very good filtering effect on strong interference signals at certain frequency.

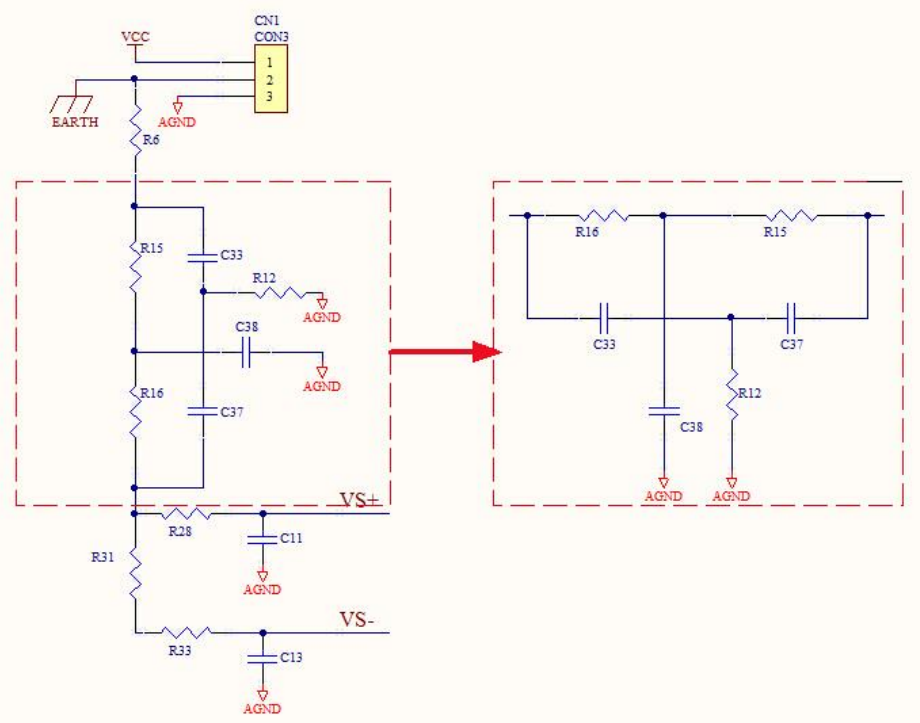

Fig.3 Double T filter

Cable insulation detection band stop filter is shown in Fig.3 which is composed of RC low pass filter and RC high pass filter. A low pass filter is composed of resistor R15, R16 and capacitor C33.It constitutes a T-network filter; high-pass filter is composed of capacitors C33, C37 and resistor R12 .It constitutes a T-network filter. Two filters are symmetrical T-shaped structure, which is called a symmetrical double-T network band-stop filter.

The double $\mathrm{T}$ filter makes use of the characteristics of capacitance that the impedance of capacitance changes with fluency. By appropriately selecting the value of capacitors and resistor, the double $\mathrm{T}$ filter can effectively select the desired frequency signal and filter out interference signals.

Assume that $\dot{U}_{i}$ is the input signal of the double T network filter and $\dot{U}_{o}$ is the output signal of the double $\mathrm{T}$ network filter. Using $\mathrm{F}$ stands for its frequency characteristic, it follows that. 
$F=\frac{\dot{U}_{o}}{\dot{U}_{i}}=\frac{s^{2}+w_{o}^{2}}{s^{2}+4 s R C+w_{o}^{2}}$

Let $s=j w$,we can get:

$$
F=\frac{1-w^{2} C^{2} R^{2}}{\left(1-w^{2} C^{2} R^{2}\right)+j 4 w R C}
$$

$$
\text { Let } w=w_{0}=\frac{1}{R C} \text {, we can get: }
$$

$$
|F|=\frac{\left|1-w^{2} C^{2} R^{2}\right|}{\sqrt{\left(1-w^{2} C^{2} R^{2}\right)^{2}+(4 w C R)^{2}}}
$$

From the above equation, it can follow that double $\mathrm{T}$ filter presents a good filtering ability for $w_{0}$ signal .In order to make use of the double T filter's characteristics filtering out interference signals from source which can have influence on the accuracy of sampling the voltage, double $\mathrm{T}$ filter is integrated into the Insulation test voltage sampling circuit.

\section{Insulation Resistance Sampling Principle}

Insulation resistance of the sampling, correction module circuit diagram is shown in Fig.4:

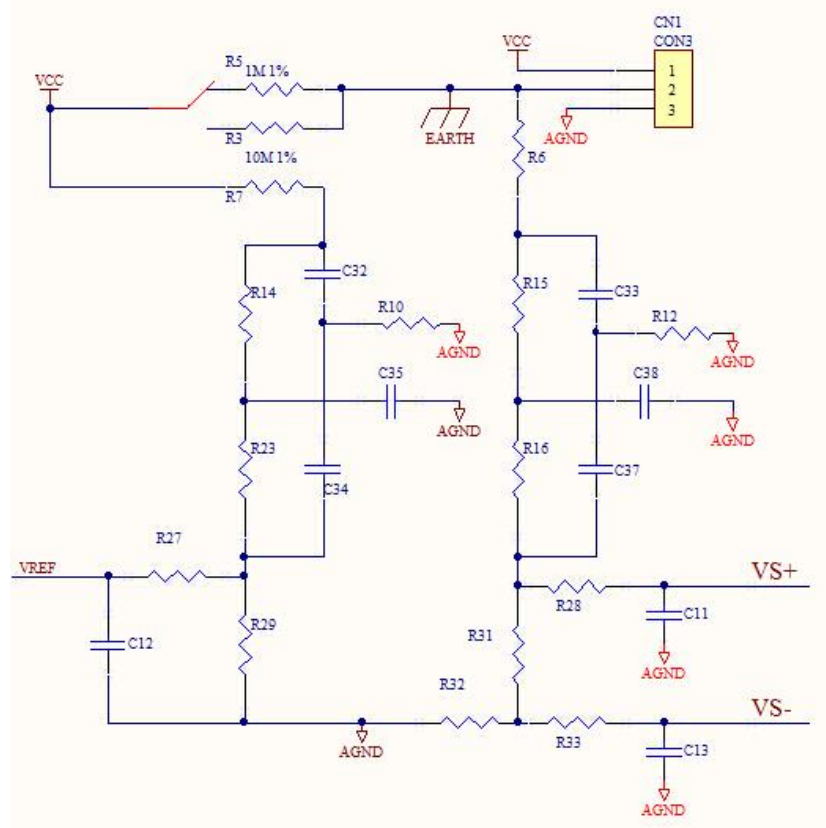

Fig.4 Insulation resistance of the sampling, correction module circuit diagram

VREF is benchmark power of ADC. $\mathrm{V}+$ and $\mathrm{V}$ - are the ADC input voltage signal (sampling voltage waited for conversion)The principle of insulation resistance measurement is stated as follows: 
By controlling relay to connect $1 \mathrm{M}$ R5 to insulation resistance sampling circuit, the R5 is measured insulation resistance.

According to the partial pressure equation we can obtain the Eq.4 and Eq.5 :

$$
\begin{aligned}
& V_{\text {in }}=V S_{+}-V S_{-}=\frac{R_{31}}{R_{5}+R_{15}+R_{24}+R_{31}+R_{32}} * V D D \\
& V R E F=\frac{R_{29}}{R_{7}+R_{14}+R_{23}+R_{29}} * V D D
\end{aligned}
$$

According to the working principle of the $\mathrm{AD}$ converter we can obtain the Eq.6:

$$
\frac{V_{i n}}{V R E F}=\frac{X_{1 M}}{2^{n}}
$$

Where, $\mathrm{n}$ stands for the resolution of the $\mathrm{AD}$ converter,

$X_{1 M}$ is the conversion result when the value of insulation resistance is $1 \mathrm{M}$. Based on the Eq.4,Eq.5 into the Eq.6,we can get:

$$
\frac{\frac{R_{31} *\left(R_{7}+R_{14}+R_{23}+R_{29}\right)}{R_{29}}}{R_{5}+\left(R_{15}+R_{24}+R_{31}+R_{32}\right)}=\frac{X_{1 M}}{2^{n}}
$$

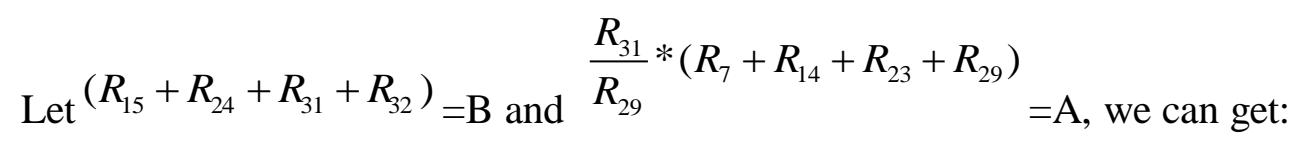

$$
\frac{2^{n} * A}{R_{5}+B}=X_{1 M}
$$

$\mathrm{A}$ and $\mathrm{B}$ are the parameter to be corrected.

In the same way ,By controlling relay to connect 10M R6 to insulation resistance sampling circuit. At present the R6 is measured insulation resistance.

Eventually we can obtain Eq.9:

$$
\frac{2^{n} * A}{R_{6}+B}=X_{10 M}
$$

Where, ${ }^{X_{10 M}}$ is the conversion result when the value of insulation resistance is $10 \mathrm{M}$. According to the Eq.8 and9we can obtain the equations group. 


$$
\frac{2^{n} * A}{R_{5}+B}=X_{1 M} \quad \frac{2^{n} * A}{R_{6}+B}=X_{10 M}
$$

Though calculating we can obtain the value of A and B.

$\mathrm{A}=A_{1}, \mathrm{~B}=B_{1}$

Finally we can get Eq.11

$$
\frac{2^{n} * A_{1}}{R_{x}+B_{1}}=X
$$

Where, $R_{x}$ is the insulation resistance of the cable under test, $X$ is the conversion result produced by $\mathrm{AD}$ converter when $R_{x}$ is the insulation resistance of the cable. Before operating electric cable insulation measurement system, system will start the correction function regularly and get the value of $\mathrm{A}$ and $\mathrm{B}$ which are needed to calculate the insulation resistance of the cable. After the correction, the correcting circuit switch is disconnected and the electric cable is connected. This algorithm can effectively reduce the influence of floating resistance fluctuating with the external environment, and largely improve the precision of the measurement of insulation resistance.

\section{The Simulation And the Result Analysis}

\section{Filter Simulation And Analysis}

In order to prove that the double $\mathrm{T}$ filter circuit is able to filter fixed frequency noise we conduct an experiment by the Multisim simulation software. Fig.5 shows the amplitude-frequency characteristic curve.

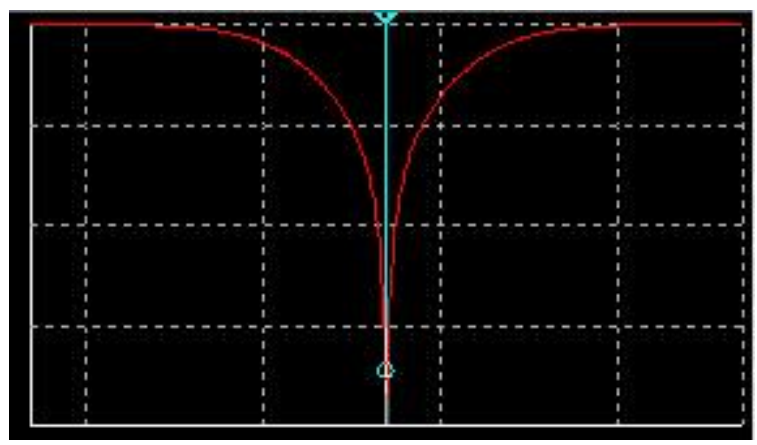

Fig.5 The amplitude frequency characteristics

After simulation by Multisim, we can get the simulation results about the amplitude-frequency characteristic curve in figure.4. Double T band-stop filter's band-stop filter characteristics perform excellent, and ,stop band is narrower. It shows the amplitude amplification of $50 \mathrm{~Hz}$ signal is $-42.972 \mathrm{~dB}$ and effectively suppress the interference produced by signal whose frequency is $50 \mathrm{~Hz}$.

\section{The Experiment of Measuring Insulation Resistance}

In the experiments, we replace the insulation resistance of the cable with testing resistance and use signal generator load interference noise to simulate the interference signal in the subway train when subway train is running.LTC2484 is used in insulation detection circuit. Power supply voltage is $400 \mathrm{~V}$. Fluke digital multimeter whose type is 187 was used in experiment for measuring and calibration standard table and its impedance measurement accuracy is $0.05 \%$. The values of testing 
resistance were $5.1 \mathrm{~K}, 10 \mathrm{~K}, 15 \mathrm{~K}, 20 \mathrm{~K}, 25 \mathrm{~K}, 50 \mathrm{~K}, 100 \mathrm{~K}, 1 \mathrm{M}, 5 \mathrm{M}$, and 10M. The experimental results are shown in table 1 and 2.

The experimental results in Table 1 was obtained by tradition insulation testing device without filtering. The results in Table 2 were obtained by insulation testing device based on double $\mathrm{T}$ tilter.

Table 1

\begin{tabular}{|l|l|l|l|l|}
\hline NO. & $\begin{array}{l}\text { Grounding } \\
\text { resistance } \\
\text { nominal } \\
\text { value / } \\
\text { K } \Omega\end{array}$ & $\begin{array}{l}\text { Grounding } \\
\text { resistance } \\
\text { measured } \\
\text { value / } \\
\text { K } \Omega\end{array}$ & $\begin{array}{l}\text { Means for } \\
\text { measuring } \\
\text { the value } \\
\text { of the / } \\
\text { K } \Omega\end{array}$ & $\begin{array}{l}\text { Relative } \\
\text { error\% }\end{array}$ \\
\hline 1 & 5.1 & 5.07 & 4.91 & 3.58 \\
\hline 2 & 10 & 10.03 & 9.53 & 4.69 \\
\hline 3 & 15 & 14.94 & 13.83 & 7.77 \\
\hline 4 & 20 & 20.15 & 18.92 & 5.39 \\
\hline 5 & 25 & 24.85 & 22.80 & 8.78 \\
\hline 6 & 50 & 49.85 & 45.91 & 8.17 \\
\hline 7 & 100 & 99.45 & 95.73 & 4.27 \\
\hline 8 & 1000 & 994.75 & 908.40 & 9.16 \\
\hline 9 & 5000 & 4969.15 & 4762 & 4.76 \\
\hline 10 & 10000 & 9939.05 & 9445 & 5.55 \\
\hline
\end{tabular}

Table 2

\begin{tabular}{|l|l|l|l|l|}
\hline NO. & $\begin{array}{l}\text { Grounding } \\
\text { resistance } \\
\text { nominal } \\
\text { value / K } \Omega\end{array}$ & $\begin{array}{l}\text { Grounding } \\
\text { resistance } \\
\text { measured } \\
\text { value / K } \Omega\end{array}$ & $\begin{array}{l}\text { Means for } \\
\text { measuring } \\
\text { the value } \\
\text { of the / } \\
\text { K } \Omega\end{array}$ & $\begin{array}{l}\text { Relative } \\
\text { error\% }\end{array}$ \\
\hline 1 & 5.1 & 5.07 & 5.00 & 1.96 \\
\hline 2 & 10 & 10.03 & 9.82 & 1.80 \\
\hline 3 & 15 & 14.94 & 14.72 & 1.86 \\
\hline 4 & 20 & 20.15 & 19.6 & 2.0 \\
\hline 5 & 25 & 24.85 & 24.51 & 1.96 \\
\hline 6 & 50 & 49.85 & 49.04 & 1.91 \\
\hline 7 & 100 & 99.45 & 98.19 & 1.81 \\
\hline 8 & 1000 & 994.75 & 981.8 & 1.82 \\
\hline 9 & 5000 & 4969.15 & 4913.5 & 1.73 \\
\hline 10 & 10000 & 9939.05 & 9833 & 1.67 \\
\hline
\end{tabular}

Through the experiment results, it follows that insulation resistance measurement error measured by tradition detection method is bigger than that measured by double $\mathrm{T}$ filter based new cable insulation detection approach. It is proved that the electromagnetic interference has a great influence on the insulation detection and can easily make the on-line insulation monitoring system unstable. We can see from table 2 that test errors remain below $2 \%$, which satisfies the requirement of subway train cable insulation level. We can draw a conclusion that double filter based new cable insulation detection approach can effectively filter electromagnetic interference and makes the equipment working in a stable state in the course of subway running. 


\section{Conclusion}

Traditional insulation detection equipment places great emphasis on the algorithm to improve detection precision ,but places little emphasis on eliminating the electromagnetic interference. Although there are many designs about shielding electromagnetic interference for every electrical module in the subway train, the cables for modules connecting are still influenced by electromagnetic interference. This paper puts forward a new scheme which combines cable insulation testing with double $\mathrm{T}$ filter. The simulation experiment proves that, double filter based new cable insulation detection approach can effectively filter electromagnetic interference and makes the equipment working in a stable state in the course of subway running and measurement error is less than $2 \%$ when conducting the sampling measurement for insulation resistance of less than $2 \mathrm{M}$,furthermore, devices can running reliably and steadily and conform to the requirements of the site.

\section{Acknowledgement}

This work is supported by the national science and technology support program major project,No.2013BAG19B01 and the project of my advisor Mr dong .

\section{Reference}

[1]WANG Cui-zhen,TANG Jin-yuan,PAN Yun-zhi. Double $\mathrm{T}$ band-stop filter network transmission characteristics research and simulation[J], Instrumentation Technology,2012(06).

[2]LIN Yankai,LIU Weidong. Antijamming Electromagnetic Interference of Substation Automation System in Subway[J]. BUILDING ELECTRICITY, 2007(05).

[3]LI Hang_sheng, XIAO Ying_chun. On-line monitoring measurement of cable insulation in microcomputer monitoring system for railway signals [J]Journal of wuhan university of hydraulic and electricengineering 2000, 33(6).

[4] Lin Yankai, EMC Design in Urban Track Mass Transit System[J], BUILDING ELECTRICITY, 2007, 26(10).

[5]LIU Shaolin. Prevention and Protective Measures against EMI of UMT Substation [J]URBAN MASS TRANSIT, 2008, 11(7).

[6]LI Zhe,GAO Bo,MA Jiajun.Design and implementation of double-T filter based on FPGA [J]Application of Electronic Technique, 2013, 39(10) 